\title{
ON STRONG INCLUSIONS AND ASYMMETRIC PROXIMITIES IN FRAMES
}

\author{
JORGE PICADO AND ALEŠ PULTR
}

\begin{abstract}
The strong inclusion, a specific type of subrelation of the order of a lattice with pseudocomplements, has been used in the concrete case of the lattice of open sets in topology for an expedient definition of proximity, and allowed for a natural point-free extension of this concept. A modification of a strong inclusion for biframes then provided a point-free model also for the non-symmetric variant. In this paper we show that a strong inclusion can be non-symmetrically modified to work directly on frames, without prior assumption of a biframe structure. The category of quasi-proximal frames thus obtained is shown to be concretely isomorphic with the biframe based one, and shown to be related to that of quasi-uniform frames in a full analogy with the symmetric case.
\end{abstract}

KEYWORDS: Frame, biframe, pseudocomplement, strong inclusion, paircover, quasiuniform frame, quasi-proximal frame, total boundedness.

AMS SubjeCt Classification (2010): 06D22, 06D15, 54E05, 54E15, 54E55.

\section{Introduction}

In a general setting, a strong inclusion is a subrelation $\triangleleft$ of the order of a lattice with pseudocomplements that

- is a sublattice of $L \times L$,

- interpolates,

- satisfies the implication

$$
a \leq b \triangleleft c \leq d \Rightarrow a \leq d,
$$

and

- $a \triangleleft b \Rightarrow b^{*} \triangleleft a^{*}$.

This concept (and term) was, first, introduced by Dowker ([4]) for purposes of enriched topology. There it naturally appears, e.g as the "completely below" relation $U \prec \prec V$ (where $U$ is separated from the complement of $V$ by

Received July 12, 2010.

Support from the Centre for Mathematics of the University of Coimbra (CMUC/FCT), project 1M0545 of the Ministry of Education of the Czech Republic and grant MTM2009-12872-C02-02 of the Ministry of Science and Innovation of Spain and FEDER is gratefully acknowledged. 
a real-valued continuous function), or " $U$ is uniformly below $V$ " in a uniform space.

In particular, the proximity, a certain enrichment of a topological structure, originally described by specifying for sets when they are "near" ("proximal" [12]) can be alternatively, and to advantage, described in terms of a strong inclusion stronger than the inclusion order.

This has proved to be particularly useful in the point-free setting where the strong inclusion, strengthening the order in a frame (a lattice with $\bigvee \wedge$ distributivity, see 1.1) makes for a suitable extension of the classical concept ([7]); imitating the "proximity of elements" makes here good sense only in (complete) Boolean algebras where it is then equivalent with the strong inclusion approach ([13]). It should be noted that besides of the properties above, the strong inclusion one deals with is further required that

- $a \triangleleft b \Rightarrow a^{*} \vee b=1$ (implying the "rather below" relation), and - for each $a, a=\bigvee\{b \mid b \triangleleft a\}$ (admissibility of the additional structure).

It turns out that the resulting category of proximity frames is concretely isomorphic to that of totally bounded uniform frames (see [7]) and that the compactifiable frames are exactly those that admit strong inclusions (see [1]).

In classical topology it was found useful to generalize proximities by dropping symmetry. This can be modeled in the point-free (frame) setting ([8], [19]) by introducing modified strong inclusions on biframes (triples $\left(L, L_{1}, L_{2}\right)$ where $L_{i}$ are specific subframes of $L$ - see 1.1 below) as couples of subrelations $\triangleleft_{i}$ of the orders of $L_{i}$ with certain intertwined properties (see 1.2). Thus generalized strong inclusions are, again, closely connected with compactifications (of biframes — see Schauerte [19]).

In our recent paper [15] we have shown, for uniformities, another environment of the frame structure, that when dropping the symmetry the biframes can be, essentially, avoided. The question naturally arises whether this can be done with the (quasi-) proximity as well. In the present paper we answer this question in the affirmative introducing (in Section 2) a category of quasi-proximal frames that enriches the plain frames directly. Such proximal structures make the picture in the point-free setting more similar to the classical one (where the bitopologies appear only a posteriori and clarify the discussion in the introduction of Doitchinov [3]). In Section 3 we prove that this category is concretely isomorphic with the biframe based one. Finally, in Section 4 the new category of quasi-proximal frames is related with that 
of quasi-uniform frames. It turns out that the relation is analogous with the symmetric case: namely, there is a one-to-one correspondence between quasi-proximities and totally bounded quasi-uniformities, yielding a concrete isomorphism of the categories.

\section{Preliminaries}

1.1. Frames and biframes. Recall that a frame is a complete lattice satisfying the distributivity law

$$
a \wedge \bigvee_{i \in I} b_{i}=\bigvee_{i \in I}\left(a \wedge b_{i}\right)
$$

and that frame homomorphisms preserve all joins (including the bottom element 0 ) and finite meets (including the top element 1). Frames and frame homomorphisms are the objects and morphisms of the category Frm.

A biframe is a triple $\left(L, L_{1}, L_{2}\right)$ in which $L$ is a frame and $L_{1}$ and $L_{2}$ are subframes of $L$ such that $L_{1} \cup L_{2}$ generates $L$ (in the sense that any element of $L$ can be expressed as a join of finite meets of elements of $L_{1} \cup L_{2}$ ); a biframe homomorphism $h:\left(L, L_{1}, L_{2}\right) \rightarrow\left(M, M_{1}, M_{2}\right)$ is a frame homomorphism from $L$ to $M$ such that the image of $L_{i}(i=1,2)$ under $h$ is contained in $M_{i}$. Biframes and biframe homomorphisms are the objects and morphisms of the category BiFrm. If $\left(L, L_{1}, L_{2}\right)$ is a biframe and $a \in L_{i}(i=1,2)$, the element

$$
a^{\bullet}=\bigvee\left\{b \in L_{j} \mid a \wedge b=0\right\} \quad(j \in\{1,2\}, j \neq i)
$$

is the analogue in biframes of the pseudocomplement

$$
a^{*}=\bigvee\{b \in L \mid a \wedge b=0\}
$$

of an element $a$ of a frame $L$.

For more about frames the reader can consult [16] or [17], for biframes see [2] and [19].

1.2. Strong inclusions on biframes: quasi-proximities. A strong inclusion [19] on a biframe $\left(L, L_{1}, L_{2}\right)$ is a pair $\left(\triangleleft_{1}, \triangleleft_{2}\right)$ of relations on $L_{1}$ and $L_{2}$ respectively satisfying the following conditions (for $i=1,2$ ):

(S1) $\triangleleft_{i}$ is a sublattice of $L_{i} \times L_{i}$.

(S2) $a \leq b \triangleleft_{i} c \leq d$ implies that $a \triangleleft_{i} d$.

(S3) $a \triangleleft_{i} b$ implies that $a^{\bullet} \vee b=1$ (usually denoted by $a \prec_{i} b$ ).

(S4) $a \triangleleft_{i} b$ implies that there exists $c \in L_{i}$ with $a \triangleleft_{i} c \triangleleft_{i} b$. 
(S5) If $a \triangleleft_{i} b$ then $b^{\bullet} \triangleleft_{j} a^{\bullet}$ for $j \in\{1,2\}$ and $j \neq i$.

(S6) For every $a \in L_{i}, a=\bigvee\left\{b \in L_{i} \mid b \triangleleft_{i} a\right\}$.

Note that the more standard strong inclusion in a frame is the $\triangleleft$ from $(\triangleleft, \triangleleft)$ on $(L, L, L)$.

A triple $\left(\left(L, L_{1}, L_{2}\right), \triangleleft_{1}, \triangleleft_{2}\right)$ where $\left(\triangleleft_{1}, \triangleleft_{2}\right)$ is a strong inclusion on the biframe $\left(L, L_{1}, L_{2}\right)$ is called a quasi-proximal frame [7] (proximal biframe in the more recent [8]). Given proximal biframes

$$
\left(\left(L, L_{1}, L_{2}\right), \triangleleft_{1}^{L}, \triangleleft_{2}^{L}\right) \quad \text { and } \quad\left(\left(M, M_{1}, M_{2}\right), \triangleleft_{1}^{M}, \triangleleft_{2}^{M}\right),
$$

a biframe homomorphism $h:\left(L, L_{1}, L_{2}\right) \rightarrow\left(M, M_{1}, M_{2}\right)$ is a proximal biframe homomorphism if

$$
a \triangleleft_{i}^{L} b \text { implies } h(a) \triangleleft_{i}^{M} h(b) \quad\left(\text { for } i=1,2 \text { and every } a, b \in L_{i}\right) .
$$

The category of proximal biframes and proximal biframe homomorphisms will be denoted by

\section{PBiFrm.}

1.3. Quasi-uniform frames. Let $L$ be a frame. A subset $C \subseteq L \times L$ is a paircover [15] of $L$ if

$$
\bigvee\left\{c_{1} \wedge c_{2} \mid\left(c_{1}, c_{2}\right) \in C\right\}=1
$$

A paircover $C$ of $L$ is strong if, for any $\left(c_{1}, c_{2}\right) \in C,\left(c_{1}, c_{2}\right)=(0,0)$ whenever $c_{1} \wedge c_{2}=0$. For any $C, D \subseteq L \times L$ we write $C \leq D$ (and say that $C$ refines $D)$ if for any $\left(c_{1}, c_{2}\right) \in C$ there is $\left(d_{1}, d_{2}\right) \in D$ with $c_{1} \leq d_{1}$ and $c_{2} \leq d_{2}$. Further we write

$$
C \wedge D=\left\{\left(c_{1} \wedge d_{1}, c_{2} \wedge d_{2}\right) \mid\left(c_{1}, c_{2}\right) \in C,\left(d_{1}, d_{2}\right) \in D\right\}
$$

obviously it is a is a paircover again.

For $a \in L$ and $C, D \subseteq L \times L$, we set

$$
\begin{aligned}
& \operatorname{st}_{1}(a, C)=\bigvee\left\{c_{1} \mid\left(c_{1}, c_{2}\right) \in C \text { and } c_{2} \wedge a \neq 0\right\}, \\
& \text { st }_{2}(a, C)=\bigvee\left\{c_{2} \mid\left(c_{1}, c_{2}\right) \in C \text { and } c_{1} \wedge a \neq 0\right\}, \\
& C^{-1}=\left\{\left(c_{2}, c_{1}\right) \mid\left(c_{1}, c_{2}\right) \in C\right\}, \text { and } \\
& \operatorname{st}(D, C)=\left\{\left(\operatorname{st}_{1}\left(d_{1}, C\right), \operatorname{st}_{2}\left(d_{2}, C\right)\right) \mid\left(d_{1}, d_{2}\right) \in D\right\}
\end{aligned}
$$

and write

$$
C^{*} \text { for } \operatorname{st}(C, C) \text {. }
$$

We shall need the following facts from [15, Proposition 2.2]: 
Proposition 1.3.1. Let $C, D \subseteq L \times L$ and $a, b \in L$. Then:

(1) If $a \leq b$ then $\operatorname{st}_{i}(a, C) \leq \operatorname{st}_{i}(b, C)$.

(2) If $C \leq D$ then $\operatorname{st}_{i}(a, C) \leq$ st $_{i}(a, D)$.

(3) $a \wedge \mathrm{st}_{1}(b, C)=0$ iff $b \wedge \mathrm{st}_{2}(a, C)=0$.

(4) If $C$ is a paircover then $a \leq \operatorname{st}_{i}(a, C)$ and $C \leq C^{*}$.

(5) If $C$ is a paircover then $\operatorname{st}_{i}\left(\operatorname{st}_{i}(a, C), C\right) \leq \operatorname{st}_{i}\left(a, C^{*}\right)$.

(6) For any frame homomorphism $h: L \rightarrow M$,

$$
\text { st }_{i}(h(a), h[C]) \leq h\left(\text { st }_{i}(a, C)\right) .
$$

(7) For any frame homomorphism $h: L \rightarrow M, h[C]^{*} \leq h\left[C^{*}\right]$.

Given a non-empty family $\mathcal{U}$ of paircovers of $L$, we write $a \triangleleft_{i}^{\mathcal{U}} b(i=1,2)$ whenever $\operatorname{st}_{i}(a, C) \leq b$ for some $C \in \mathcal{U}$, and set

$$
L_{i}(\mathcal{U})=\left\{a \in L \mid a=\bigvee\left\{b \in L \mid b \triangleleft_{i}^{\mathcal{U}} a\right\}\right\} \quad(i=1,2) .
$$

From [15, Proposition 2.4] we know the following:

Proposition 1.3.2. Let $\mathcal{U}$ be a basis for a filter of paircovers of $L$. Then, for $i=1,2$ :

(1) The relations $\triangleleft_{i}^{\mathcal{U}}$ are sublattices of $L \times L$, both stronger than $\leq$.

(2) For any $a, b, c, d \in L, a \leq b \triangleleft_{i}^{\mathcal{U}} c \leq d$ implies $a \triangleleft_{i}^{\mathcal{U}} d$.

(3) $L_{i}(\mathcal{U})$ are subframes of $L$.

A system $\mathcal{U}$ of paircovers of $L$ is admissible [15] if $\left(L, L_{1}(\mathcal{U}), L_{2}(\mathcal{U})\right)$ is a biframe or, equivalently, if for every $a \in L, a=\bigvee\left\{b \in L \mid b \triangleleft_{i}^{\overline{\mathcal{U}}} a\right\}$, where $\overline{\mathcal{U}}$ denotes the filter of paircovers of $L$ generated by $\left\{C \wedge C^{-1} \mid C \in \mathcal{U}\right\}$.

Now, an admissible system $\mathcal{U}$ of paircovers of $L$ is a quasi-uniformity on $L$ if it satisfies the following conditions:

(QU1) For any $C \in \mathcal{U}$ and any paircover $D$ with $C \leq D$, then $D \in \mathcal{U}$.

(QU2) For any $C, D \in \mathcal{U}$ there exists a strong $E \in \mathcal{U}$ such that $E \leq C \wedge D$.

(QU3) For any $C \in \mathcal{U}$ there is a $D \in \mathcal{U}$ such that $D^{*} \leq C$.

The pair $(L, \mathcal{U})$ is called a quasi-uniform frame [15]. Let $(L, \mathcal{U})$ and $(M, \mathcal{V})$ be quasi-uniform frames. A frame homomorphism $h: L \rightarrow M$ is uniform if $h[C] \in \mathcal{V}$ for every $C \in \mathcal{U}$. The resulting category will be denoted by

\section{QUFrm.}

We say that a quasi-uniform frame $(L, \mathcal{U})$ is totally bounded if for every $C \in \mathcal{U}$ there is a finite paircover $D \in \mathcal{U}$ such that $D \leq C$. 
Quasi-uniform frames $(L, \mathcal{U})$ have the following crucial properties (see [15, lemmata 2.6, 3.2, 3.4]):

Consider the interior operator on $\mathcal{P}(L \times L)$ defined by

$$
\operatorname{int}(C)=\bigcup\{D \subseteq L \times L \mid D \stackrel{\mathcal{U}}{\subseteq} C\},
$$

where

$$
C \stackrel{\mathcal{U}}{\Subset} D \equiv_{\text {def }} \operatorname{st}(C, U) \leq D \text { for some } U \in \mathcal{U}
$$

Proposition 1.3.3. For every $C \in \mathcal{U}$ we have:

(1) $\operatorname{int}(C) \leq C \leq \operatorname{int}\left(C^{*}\right)$.

(2) $\operatorname{st}_{i}(a, \operatorname{int}(C)) \in L_{i}(\mathcal{U})(i=1,2)$ for every $a \in L$.

Given $U \in \mathcal{U}, a \in L$ is said to be $U$-small if

$$
a \leq \bigvee\left\{u_{1} \wedge u_{2} \mid\left(u_{1}, u_{2}\right) \in U, u_{1} \wedge u_{2} \wedge b \neq 0\right\}
$$

whenever $a \wedge b \neq 0$. Let

$$
C_{U}=\left\{\left(\operatorname{st}_{1}(a, \operatorname{int}(U)), \operatorname{st}_{2}(a, \operatorname{int}(U))\right) \mid a \text { is an } U \text {-small member of } L\right\} .
$$

Proposition 1.3.4. (1) Each $C_{U}$ is a strong paircover of $L$ contained in $L_{1}(\mathcal{U}) \times L_{2}(\mathcal{U})$.

(2) $\operatorname{st}_{i}\left(a, C_{U}\right) \leq \operatorname{st}_{i}\left(a, U^{* *}\right)(i=1,2)$.

(3) $\mathrm{st}_{i}(a, U) \leq \operatorname{st}_{i}\left(a, C_{U^{*}}\right)(i=1,2)$.

(4) $C_{U} \leq U^{* *}$; if $U$ is strong then $U \leq C_{U^{*}}$.

\section{Quasi-proximities without biframes}

Let $L$ be a frame, $\triangleleft$ a binary relation in $L$ and

$$
L(\triangleleft)=\{a \in L \mid a=\bigvee\{b \in L \mid b \triangleleft a\}\} .
$$

Lemma 2.1. If $\triangleleft$ is a sublattice of $L \times L$, stronger than $\leq$ (that is, $\triangleleft \subseteq \leq$ ), satisfying

$$
a \leq b \triangleleft c \leq d \Rightarrow a \triangleleft d
$$

then $L(\triangleleft)$ is a subframe of $L$. 
Proof: Since $0 \triangleleft 0$ and $1 \triangleleft 1$, then $0,1 \in L(\triangleleft)$. Since $\triangleleft \subseteq \leq$, we have $\bigvee\{b \in L \mid b \triangleleft a\} \leq a$. Let $a, b \in L(\triangleleft)$. Then, since $\triangleleft$ is closed under finite meets,

$$
\begin{aligned}
a \wedge b & =\bigvee\left\{a^{\prime} \in L \mid a^{\prime} \triangleleft a\right\} \wedge \bigvee\left\{b^{\prime} \in L \mid b^{\prime} \triangleleft b\right\}= \\
& =\bigvee\left\{a^{\prime} \wedge b^{\prime} \mid a^{\prime}, b^{\prime} \in L, a^{\prime} \triangleleft a, b^{\prime} \triangleleft b\right\} \leq \bigvee\{c \in L \mid c \triangleleft a \wedge b\}
\end{aligned}
$$

which shows that $a \wedge b \in L(\triangleleft)$.

Now, let $a_{i} \in L(\triangleleft)(i \in I)$. Then $\bigvee_{i \in I} a_{i}=\bigvee_{i \in I} \bigvee\left\{b \in L \mid b \triangleleft a_{i}\right\}$. For each such $b, b \triangleleft a_{i} \leq \bigvee_{i \in I} a_{i}$. Consequently, by (2.1.1), $\bigvee_{i \in I} a_{i} \leq \bigvee\{b \in L \mid$ $\left.b \triangleleft \bigvee_{i \in I} a_{i}\right\}$ and $\bigvee_{i \in I} a_{i} \in L(\triangleleft)$.

In the sequel we will have to refer to pseudocomplements relatively to distinct subframes. Therefore we will adopt the following notation: for a subframe $K$ of a frame $L$ and $a \in L$, we denote by $\mathrm{c}_{K}(a)$ the element

$$
\bigvee\{b \in K \mid b \wedge a=0\} .
$$

We have $\mathrm{c}_{L}(a)=a^{*}, \mathrm{c}_{K}(a) \leq a^{*}, \mathrm{c}_{K}(0)=1, \mathrm{c}_{K}(1)=0$ and $a \leq \mathrm{c}_{K}\left(\mathrm{c}_{K}(a)\right)$ for every $a \in K$.

Definition 2.2. Let $L$ be a frame. A pair $\left(\triangleleft_{1}, \triangleleft_{2}\right)$ of relations in $L$ will be called a strong bi-inclusion on $L$ if for $i, j \in\{1,2\}$ we have:

(SB1) $\triangleleft_{i}$ is a sublattice of $L \times L$.

(SB2) $a \leq b \triangleleft_{i} c \leq d$ implies that $a \triangleleft_{i} d$.

(SB3) $a \triangleleft_{i} b$ implies that $\mathrm{c}_{L\left(\triangleleft_{j}\right)}(a) \vee b=1(j \neq i)$.

(SB4) $a \triangleleft_{i} b$ implies that there exists $c \in L\left(\triangleleft_{i}\right)$ with $a \triangleleft_{i} c \triangleleft_{i} b$.

(SB5) If $a \triangleleft_{i} b$ then $b^{*} \triangleleft_{j} a^{*}$ for $j \neq i$.

(SB6) $\left(L, L\left(\triangleleft_{1}\right), L\left(\triangleleft_{2}\right)\right)$ is a biframe.

Remarks 2.3. (1) (SB3) implies that each $\triangleleft_{i}(i=1,2)$ is stronger than $\leq$ : if $\mathrm{c}_{L\left(\triangleleft_{j}\right)}(a) \vee b=1$ then $a=a \wedge\left(\mathrm{c}_{L\left(\triangleleft_{j}\right)}(a) \vee b\right)=a \wedge b$. It also implies that $b^{*} \leq \mathrm{c}_{L\left(\triangleleft_{j}\right)}(a)$ whenever $a \triangleleft_{i} b$ (because $\mathrm{c}_{L\left(\triangleleft_{j}\right)}(a) \vee b=1$ and $b^{*} \wedge b=0$ ).

(2) For any $a, b \in L$,

$$
a \triangleleft_{i} b^{*} \Rightarrow a \triangleleft_{i} \mathrm{c}_{L\left(\triangleleft_{i}\right)}(b) \quad(i=1,2) .
$$

Indeed, by (SB4) there exists $c \in L\left(\triangleleft_{i}\right)$ such that $a \triangleleft_{i} c \triangleleft_{i} b^{*}$. Since $c \leq b^{*}$, then $c \wedge b=0$ and consequently $c \leq \mathrm{c}_{L\left(\triangleleft_{i}\right)}(b)$. Therefore $a \triangleleft_{i} \mathrm{c}_{L\left(\triangleleft_{i}\right)}(b)$ by (SB2). 
(3) Also, it may be worth mentioning that any pair $\left(\triangleleft_{1}, \triangleleft_{2}\right)$ satisfying (SB2), (SB3) and (SB4) satisfies (SB5) if and only if it satisfies

(SB5a) if $a \triangleleft_{i} b$ then $\mathrm{c}_{L\left(\triangleleft_{j}\right)}(b) \triangleleft_{j} \mathrm{c}_{L\left(\triangleleft_{j}\right)}(a)$ for $j \neq i$.

Indeed:

$\Rightarrow$ : If $a \triangleleft_{i} b$ then, by (SB5), $\mathrm{c}_{L\left(\triangleleft_{j}\right)}(b) \leq b^{*} \triangleleft_{j} a^{*}$ and therefore by the preceding remark $\mathrm{c}_{L\left(\triangleleft_{j}\right)}(b) \triangleleft_{j} \mathrm{c}_{L\left(\triangleleft_{j}\right)}(a)$.

$\Leftarrow$ : In order to prove (SB5), suppose $a \triangleleft_{i} b$ and apply (SB4) to get $c$ satisfying $a \triangleleft_{i} c \triangleleft_{i} b$. By (SB5a) and Remark (1) we obtain $b^{*} \leq \mathrm{c}_{L\left(\triangleleft_{j}\right)}(b) \triangleleft_{j} \mathrm{c}_{L\left(\triangleleft_{j}\right)}(a) \leq$ $a^{*}$.

(4) (SB3) is obviously stronger than

(SB3a) $a \triangleleft_{i} b$ implies that $a^{*} \vee b=1$

(since $\left.\mathrm{c}_{L\left(\triangleleft_{j}\right)}(a) \leq a^{*}\right)$. However, if $\left(\triangleleft_{1}, \triangleleft_{2}\right)$ satisfies (SB2) and (SB4) then it satisfies (SB3) and (SB5) if and only if it satisfies (SB3a) and (SB5a): the implication " $\Rightarrow$ " was already proved in (3) and, conversely, if $a \triangleleft_{i} b$ then, by (SB5a), $\mathrm{c}_{L\left(\triangleleft_{j}\right)}(b) \triangleleft_{j} \quad \mathrm{c}_{L\left(\triangleleft_{j}\right)}(a)$; applying (SB3a) we get $\left(\mathrm{c}_{L\left(\triangleleft_{j}\right)}(b)\right)^{*} \vee$ $\mathrm{c}_{L\left(\triangleleft_{j}\right)}(a)=1$ and thus $b \vee \mathrm{c}_{L\left(\triangleleft_{j}\right)}(a)=1\left(\right.$ since $\left.b \leq\left(\mathrm{c}_{L\left(\triangleleft_{j}\right)}(b)\right)^{*}\right)$.

(5) In addition, (SB3) may be equivalently replaced by the conjunction (SB0) for every $a, b \in L, a \triangleleft_{i} b \Rightarrow a \leq b$, and (SB3b) for every $a, b \in L\left(\triangleleft_{i}\right), a \triangleleft_{i} b$ implies that $\mathrm{c}_{L\left(\triangleleft_{j}\right)}(a) \vee b=1(j \neq i)$.

Indeed, the implication

$$
(\mathrm{SB} 3) \Rightarrow(\mathrm{SB} 0)+(\mathrm{SB} 3 \mathrm{~b})
$$

is obvious by the preceding observation. On the other hand, if $a \triangleleft_{i} b$ then there exist by (SB4) $c, d \in L\left(\triangleleft_{i}\right)$ such that $a \triangleleft_{i} c \triangleleft_{i} d \triangleleft_{i} b$. Thus, by (SB3b), $\mathrm{c}_{L\left(\triangleleft_{j}\right)}(c) \vee d=1$. But $d \leq b$ and $\mathrm{c}_{L\left(\triangleleft_{j}\right)}(c) \leq \mathrm{c}_{L\left(\triangleleft_{j}\right)}(a)$ (because $a \leq c$ ) and hence $\mathrm{c}_{L\left(\triangleleft_{j}\right)}(a) \vee b=1$.

Any strong bi-inclusion $\left(\triangleleft_{1}, \triangleleft_{2}\right)$ on a frame $L$ induces two subframes $L\left(\triangleleft_{1}\right)$ and $L\left(\triangleleft_{2}\right)$ of $L$. The triple $\left(L, L\left(\triangleleft_{1}\right), L\left(\triangleleft_{2}\right)\right)$ is a biframe by (SB6). The following proposition provides an alternative to condition (SB6) that avoids biframes. 
Proposition 2.4. Let $\left(\triangleleft_{1}, \triangleleft_{2}\right)$ be a pair of binary relations in $L$, both stronger than $\leq$ and satisfying (SB1) and (SB2). Then $\left(\triangleleft_{1}, \triangleleft_{2}\right)$ satisfies (SB6) if and only if

$$
\left(\mathrm{SB}^{\prime}\right) \text { for each } a \in L, a=\bigvee\{b \in L \mid b \triangleleft a\},
$$

where $\triangleleft$ is the binary relation in $L$ defined by

$$
b \triangleleft a \equiv_{\text {def }} \exists a_{1} \in L\left(\triangleleft_{1}\right), \exists a_{2} \in L\left(\triangleleft_{2}\right), a_{1} \wedge a_{2} \leq a, b \triangleleft_{1} a_{1} \text { and } b \triangleleft_{2} a_{2} .
$$

Proof: $\Rightarrow$ : For each $a \in L$ we can write $a=\bigvee_{i \in I}\left(a_{i}^{1} \wedge a_{i}^{2}\right)$ for some

$$
\left\{a_{i}^{1} \mid i \in I\right\} \subseteq L\left(\triangleleft_{1}\right) \quad \text { and } \quad\left\{a_{i}^{2} \mid i \in I\right\} \subseteq L\left(\triangleleft_{2}\right) .
$$

Taking into account that, for any $i \in I$,

$$
a_{i}^{1}=\left\{b \in L \mid b \triangleleft_{1} a_{i}^{1}\right\} \quad \text { and } \quad a_{i}^{2}=\left\{b \in L \mid b \triangleleft_{2} a_{i}^{2}\right\},
$$

it suffices to show that $b_{1} \wedge b_{2} \triangleleft a_{1} \wedge a_{2}$ whenever $b_{1} \triangleleft_{1} a_{1}$ and $b_{2} \triangleleft_{2} a_{2}$. This, however, is an immediate consequence of (SB1) and the definition of $\triangleleft$.

$\Leftarrow$ : By 2.1, each $L\left(\triangleleft_{i}\right)(i=1,2)$ is a subframe of $L$. It remains to show that each $a \in L$ is a join of finite meets in $L\left(\triangleleft_{1}\right) \cup L\left(\triangleleft_{2}\right)$.

Let $a \in L$. Then $a=\bigvee S$ where $S=\{b \in L \mid b \triangleleft a\}$. For each $b \in S$ there exist $a_{1}^{b} \in L\left(\triangleleft_{1}\right)$ and $a_{2}^{b} \in L\left(\triangleleft_{2}\right)$ satisfying $b \triangleleft_{1} a_{1}^{b}, b \triangleleft_{2} a_{2}^{b}$ and $a_{1}^{b} \wedge a_{2}^{b} \leq a$. Hence $a=\bigvee_{b \in S} b \leq \bigvee_{b \in S}\left(a_{1}^{b} \wedge a_{2}^{b}\right) \leq a$.

A frame $L$ with a strong bi-inclusion $\left(\triangleleft_{1}, \triangleleft_{2}\right)$ will be called a quasi-proximal frame. Given quasi-proximal frames $\left(L, \triangleleft_{1}^{L}, \triangleleft_{2}^{L}\right)$ and $\left(M, \triangleleft_{1}^{M}, \triangleleft_{2}^{M}\right)$, a quasiproximal map

$$
h:\left(L, \triangleleft_{1}^{L}, \triangleleft_{2}^{L}\right) \rightarrow\left(M, \triangleleft_{1}^{M}, \triangleleft_{2}^{M}\right)
$$

is a frame homomorphism $h: L \rightarrow M$ such that $a \triangleleft_{i}^{L} b \Rightarrow h(a) \triangleleft_{i}^{M} h(b)$ for every $a, b \in L$. The resulting category will be denoted by

\section{QPFrm.}

Note that our definition of a quasi-proximal frame contains, of course, the symmetric case of proximal frames (as defined by strong inclusions) [7]: it is a frame equipped with a strong bi-inclusion $\left(\triangleleft_{1}, \triangleleft_{2}\right)$ such that $\triangleleft_{1}=\triangleleft_{2}$.

Quasi-uniform frames provide canonical examples of quasi-proximal frames:

Proposition 2.5. For each quasi-uniform frame $(L, \mathcal{U})$, the triple $\left(L, \triangleleft_{1}^{\mathcal{U}}, \triangleleft_{2}^{\mathcal{U}}\right)$ is a quasi-proximal frame. 
Proof: The properties (SB1) and (SB2) follow from Proposition 1.3.2, and (SB6) is obvious.

(SB3) Suppose $a \triangleleft_{i}^{\mathcal{U}} b$. Then $\mathrm{st}_{i}(a, U) \leq b$ for some $U \in \mathcal{U}$. It suffices to show that $\mathrm{c}_{L\left(\triangleleft_{j}\right)}(a) \vee \mathrm{st}_{i}(a, U)=1$. Consider $V \in \mathcal{U}$ such that $V^{* * *} \leq U$. By Proposition 1.3.4, $V \leq C_{V^{*}}$ and $C_{V^{*}} \leq V^{* * *} \leq U$. Therefore $\operatorname{st}_{i}\left(a, C_{V^{*}}\right) \leq$ $\mathrm{st}_{i}(a, U)$. Let

$$
\left(v_{1}, v_{2}\right) \in C_{V^{*}} \subseteq L_{1}(\mathcal{U}) \times L_{2}(\mathcal{U}) .
$$

If $u_{j} \wedge a=0$ then $u_{j} \leq \mathrm{c}_{L\left(\triangleleft_{j}\right)}(a)$; otherwise, $u_{i} \leq \operatorname{st}_{i}\left(a, C_{V^{*}}\right) \leq \operatorname{st}_{i}(a, U)$. This shows that

$$
\bigvee\left\{u_{1} \wedge u_{2} \mid\left(u_{1}, u_{2}\right) \in C_{V^{*}}\right\} \leq \mathrm{c}_{L\left(\triangleleft_{j}\right)}(a) \vee \operatorname{st}_{i}(a, U)
$$

Hence $\mathrm{c}_{L\left(\triangleleft_{j}\right)}(a) \vee \mathrm{st}_{i}(a, U)=1$, since $C_{V^{*}}$ is a paircover.

(SB4) Let $\operatorname{st}_{i}(a, U) \leq b$ for some $U \in \mathcal{U}$ and take $V \in \mathcal{U}$ such that $V^{* *} \leq U$. By Proposition 1.3.3,

$$
\operatorname{st}_{i}\left(a, \operatorname{int}\left(V^{*}\right)\right) \in L_{i}(\mathcal{U})=L_{\triangleleft_{i}^{\mathcal{u}}} .
$$

Of course $a \triangleleft_{i}^{\mathcal{U}} \operatorname{st}_{i}\left(a, \operatorname{int}\left(V^{*}\right)\right)$. On the other hand, by 1.3.1(5),

$$
\left.\left.\mathrm{st}_{i}\left(\operatorname{st}_{i}\left(a, \operatorname{int}\left(V^{*}\right)\right), V^{*}\right)\right) \leq \operatorname{st}_{i}\left(\operatorname{st}_{i}\left(a, V^{*}\right), V^{*}\right)\right) \leq \operatorname{st}_{i}\left(a, V^{* *}\right) \operatorname{st}_{i}(a, U) \leq b
$$

and hence $\operatorname{st}_{i}\left(a, \operatorname{int}\left(V^{*}\right)\right) \triangleleft_{i}^{\mathcal{U}} b$.

(SB5) Let $\operatorname{st}_{i}(a, U) \leq b$ for some $U \in \mathcal{U}$. Then $\operatorname{st}_{i}(a, U) \wedge b^{*}=0(j \neq i)$. By 1.3.1(3), $a \wedge \mathrm{st}_{j}\left(b^{*}, U\right)=0$, that is, $\mathrm{st}_{j}\left(b^{*}, U\right) \leq a^{*}$.

\section{The concrete isomorphism QPFrm $\cong$ QPBiFrm}

Given a proximal biframe $\left(\left(L, L_{1}, L_{2}\right), \triangleleft_{1}, \triangleleft_{2}\right)$, let

$$
\Phi\left(\left(L, L_{1}, L_{2}\right), \triangleleft_{1}, \triangleleft_{2}\right)=\left(L, \bar{\triangleleft}_{1}, \bar{\triangleleft}_{2}\right)
$$

where, for any $a, b \in L$,

$$
a \bar{\triangleleft}_{i} b \equiv_{\text {def }} \exists c, d \in L_{i}: a \leq c \triangleleft_{i} d \leq b \quad(i=1,2) .
$$

Proposition 3.1. For any proximal biframe $\left(\left(L, L_{1}, L_{2}\right), \triangleleft_{1}, \triangleleft_{2}\right)$,

$$
\Phi\left(\left(L, L_{1}, L_{2}\right), \triangleleft_{1}, \triangleleft_{2}\right)
$$

is a quasi-proximal frame. 
Proof: (SB1) $0 \bar{\triangleleft}_{i} 0$ and $1 \bar{\triangleleft}_{i} 1$ are trivial. Let $a_{1}, a_{2} \bar{\triangleleft}_{i} b$. Then $a_{1} \leq c_{1} \triangleleft_{i}$ $d_{1} \leq b$ and $a_{2} \leq c_{2} \triangleleft_{i} d_{2} \leq b$ for some $c_{1}, c_{2}, d_{1}, d_{2} \in L_{i}$. Consequently, $a_{1} \leq c_{1} \triangleleft_{i} d_{1} \vee d_{2} \leq b$ and $a_{2} \leq c_{2} \triangleleft_{i} d_{1} \vee d_{2} \leq b$ with $c_{1}, c_{2}, d_{1} \vee d_{2} \in L_{i}$. By hypothesis, $c_{1} \wedge c_{2} \triangleleft_{i} d_{1} \vee d_{2}$ and $c_{1} \wedge c_{2} \in L_{i}$. Thus $a_{1} \wedge a_{2} \bar{\triangleleft}_{i} b$. Similarly, $a_{1} \vee a_{2} \leq c_{1} \vee c_{2} \triangleleft_{i} d_{1} \vee d_{2} \leq b$ and $a_{1} \vee a_{2} \bar{\triangleleft}_{i} b$.

(SB2) is obvious.

(SB3) First note that $L_{i} \subseteq L\left(\bar{\triangleleft}_{i}\right)$. Indeed, for each $a \in L_{i}$, since $\triangleleft_{i} \subseteq \bar{\triangleleft}_{i}$, we have

$$
a=\bigvee\left\{b \in L_{i} \mid b \triangleleft_{i} a\right\} \leq \bigvee\left\{b \in L \mid b \bar{\triangleleft}_{i} a\right\} \leq a
$$

Now, let $a, b \in L\left(\bar{\triangleleft}_{i}\right)$ and $a \leq c \triangleleft_{i} d \leq b$ with $c, d \in L_{i}$. By hypothesis, $\mathrm{c}_{L\left(\triangleleft_{j}\right)}(c) \vee d=1$ and from the inclusion $L_{i} \subseteq L\left(\bar{\triangleleft}_{i}\right)$ and the fact that $a \leq c$ it follows that

$\mathrm{c}_{L\left(\bar{\triangleleft}_{j}\right)}(a)=\bigvee\left\{a^{\prime} \in L\left(\bar{\triangleleft}_{j}\right) \mid a^{\prime} \wedge a=0\right\} \geq \bigvee\left\{c^{\prime} \in L_{j} \mid c^{\prime} \wedge c=0\right\}=\mathrm{c}_{L\left(\triangleleft_{j}\right)}(c)$.

Hence $c_{L\left(\bar{\triangleleft}_{j}\right)}(a) \vee b=1$.

(SB4) follows immediately from (S4) and the fact that $L_{i} \subseteq L\left(\bar{\triangleleft}_{i}\right)$ proved above.

(SB5) By Remark 2.3(3) it suffices to prove (SB5a). Let $a, b \in L$ with $a \varangle_{i} b$, that is, $a \leq c \triangleleft_{i} d \leq b$ for some $c, d \in L_{i}$. Then, by hypothesis, $\mathrm{c}_{L\left(\triangleleft_{j}\right)}(d) \triangleleft_{j} \mathrm{c}_{L\left(\triangleleft_{j}\right)}(c)$ and, of course, $\mathrm{c}_{L\left(\triangleleft_{j}\right)}(d), \mathrm{c}_{L\left(\triangleleft_{j}\right)}(c) \in L_{j}$. Now it suffices to show that $\mathrm{c}_{L\left(\bar{\triangleleft}_{j}\right)}(b) \leq \mathrm{c}_{L\left(\triangleleft_{j}\right)}(d)$ and $\mathrm{c}_{L\left(\triangleleft_{j}\right)}(c) \leq \mathrm{c}_{L\left(\bar{\triangleleft}_{j}\right)}(a)$. The latter was already proved in (SB3) above and the former can be proved in a similar way.

(SB6) By Lemma 2.1, each $L\left(\bar{\triangleleft}_{i}\right)$ is a subframe of $L$. Since $\left(L, L_{1}, L_{2}\right)$ is a biframe and $L_{i} \subseteq L\left(\bar{\triangleleft}_{i}\right)(i=1,2)$, then immediately $\left(L, L\left(\bar{\triangleleft}_{1}\right), L\left(\bar{\triangleleft}_{2}\right)\right)$ is also a biframe.

Given a quasi-proximal frame $\left(L, \triangleleft_{1}, \triangleleft_{2}\right)$, let

$$
\Psi\left(L, \triangleleft_{1}, \triangleleft_{2}\right)=\left(\left(L, L\left(\triangleleft_{1}\right), L\left(\triangleleft_{2}\right)\right),\left.\triangleleft_{1}\right|_{L\left(\triangleleft_{1}\right)},\left.\triangleleft_{2}\right|_{L\left(\triangleleft_{2}\right)}\right) .
$$

Proposition 3.2. For any quasi-proximal frame $\left(L, \triangleleft_{1}, \triangleleft_{2}\right), \Psi\left(L, \triangleleft_{1}, \triangleleft_{2}\right)$ is a proximal biframe.

Proof: By hypothesis, $\left(L, L\left(\triangleleft_{1}\right), L\left(\triangleleft_{2}\right)\right)$ is a biframe and $\Psi\left(L, \triangleleft_{1}, \triangleleft_{2}\right)$ satisfies conditions (S1)-(S5) trivially. It remains to check (S6): 
For every $a \in L\left(\triangleleft_{i}\right), a=\bigvee\left\{b \in L \mid b \triangleleft_{i} a\right\}$. But by condition (SB4) there is some $c \in L\left(\triangleleft_{i}\right)$ satisfying $b \triangleleft_{i} c \triangleleft_{i} a$. Therefore $a=\bigvee\left\{b \in L\left(\triangleleft_{i}\right) \mid b \triangleleft_{i} a\right\}$, as desired.

Concerning morphisms, the next result allows us to define $\Phi(h)=h$ for every $h \in$ PBiFrm and $\Psi(h)=h$ for every $h \in$ QPFrm.

Proposition 3.3. (1) Let $h:\left(\left(L, L_{1}, L_{2}\right), \triangleleft_{1}^{L}, \triangleleft_{2}^{L}\right) \rightarrow\left(\left(M, M_{1}, M_{2}\right), \triangleleft_{1}^{M}, \triangleleft_{2}^{M}\right)$ be a proximal biframe homomorphism. Then

$$
h: \Phi\left(\left(L, L_{1}, L_{2}\right), \triangleleft_{1}^{L}, \triangleleft_{2}^{L}\right) \rightarrow \Phi\left(\left(M, M_{1}, M_{2}\right), \triangleleft_{1}^{M}, \triangleleft_{2}^{M}\right) \in \text { QPFrm. }
$$

(2) Let $h:\left(L, \triangleleft_{1}^{L}, \triangleleft_{2}^{L}\right) \rightarrow\left(M, \triangleleft_{1}^{M}, \triangleleft_{2}^{M}\right)$ be a quasi-proximal map. Then

$$
h: \Psi\left(L, \triangleleft_{1}^{L}, \triangleleft_{2}^{L}\right) \rightarrow \Psi\left(M, \triangleleft_{1}^{M}, \triangleleft_{2}^{M}\right) \in \text { PBiFrm. }
$$

Proof: (1) We have to check that

$$
\forall a, b \in L_{i}, a \bar{\triangleleft}_{i}^{L} b \Rightarrow h(a) \bar{\triangleleft}_{i}^{M} h(b) \quad(i=1,2) .
$$

Let $a \triangleleft_{i}^{L} b$, that is $a \leq c \triangleleft_{i}^{L} d \leq b$ for some $c, d \in L_{i}$. Then, by hypothesis, $h(c), h(d) \in M_{i}$ and $h(a) \leq h(c) \triangleleft_{i}^{M} h(d) \leq h(b)$, which shows that $h(a) \bar{\triangleleft}_{i}^{M} h(b)$.

(2) It suffices to check that $h$ is a biframe map

$$
\left(L, L\left(\triangleleft_{1}^{L}\right), L\left(\triangleleft_{2}^{L}\right)\right) \rightarrow\left(M, M\left(\triangleleft_{1}^{M}\right), M\left(\triangleleft_{2}^{M}\right)\right)
$$

(the rest is obvious). Consider $a \in L\left(\triangleleft_{i}^{L}\right)$. Since $a=\bigvee\left\{b \in L \mid b \triangleleft_{i}^{L} a\right\}$ and $b \triangleleft_{i}^{L} a$ implies $h(b) \triangleleft_{i}^{M} h(a)$, then

$$
h(a)=\bigvee\left\{h(b) \mid b \in L, b \triangleleft_{i}^{L} a\right\} \leq \bigvee\left\{c \in M \mid c \triangleleft_{i}^{M} h(a)\right\} \leq h(a) .
$$

Hence $h(a) \in M\left(\triangleleft_{i}^{M}\right)$.

Finally, we have:

Theorem 3.4. The functors $\Psi$ and $\Phi$ constitute a concrete isomorphism between QPFrm and PBiFrm.

Proof: It suffices to show that

$$
\text { (a) } \Phi \Psi=\operatorname{Id}_{Q P F r m} \text { and (b) } \Psi \Phi=I_{\text {PBiFrm }}
$$

on objects.

(a) We will show that $\left.\varangle\right|_{L\left(\triangleleft_{i}\right)}=\triangleleft_{i}$. Consider $a, b \in L$ with $a \triangleleft_{i} b$. By (SB4), there is $c, d \in L\left(\triangleleft_{i}\right)$ such that $a \triangleleft_{i} c \triangleleft_{i} d \triangleleft_{i} b$. Since $\left.c \triangleleft_{i}\right|_{L\left(\triangleleft_{i}\right)} d$ 
then, immediately, $\left.a \triangleleft\right|_{L\left(\triangleleft_{i}\right)} b$. On the other hand, if $a, b \in L$ are such that $\left.a \bar{\triangleleft}\right|_{L\left(\triangleleft_{i}\right)} b$ then there exists a pair $c, d$ of elements of $L\left(\triangleleft_{i}\right)$ satisfying $a \leq c \triangleleft_{i} d \leq b$. Thus $a \triangleleft_{i} b$.

(b) It suffices to check that $L\left(\bar{\triangleleft}_{i}\right)=L_{i}$ for $i=1,2$. Let $a \in L_{i}$. Then, since $\triangleleft_{i} \subseteq \bar{\triangleleft}_{i}$, we have

$$
a=\bigvee\left\{b \in L_{i} \mid b \triangleleft_{i} a\right\} \leq \bigvee\left\{b \in L \mid b \bar{\triangleleft}_{i} a\right\} \leq a .
$$

Conversely, if $a \in L\left(\bar{\triangleleft}_{i}\right)$, meaning that $a \in L$ and $a=\bigvee\left\{b \in L \mid b \bar{\triangleleft}_{i} a\right\}$, then for each such $b$ there is $c_{b}, d_{b} \in L_{i}$ satisfying $b \leq c_{b} \triangleleft_{i} d_{b} \leq a$. Consequently,

$$
a \leq \bigvee\left\{c \in L_{i} \mid c \triangleleft_{i} a\right\} \leq a
$$

and, therefore, $a \in L_{i}$.

Remark 3.5. As a consequence of Proposition 1 of Schauerte [19], every quasi-proximity $\left(\triangleleft_{1}, \triangleleft_{2}\right)$ on a frame $L$ induces a compactification of the associated biframe $\left(L, L\left(\triangleleft_{1}\right), L\left(\triangleleft_{2}\right)\right)$. Then by Proposition 3 of [19] this is a zero-dimensional compactification if and only if $a \triangleleft_{i} b$ (for $a, b \in L\left(\triangleleft_{i}\right.$ ), $i=1,2)$ implies the existence of $c \in L\left(\triangleleft_{i}\right)$ satisfying $a \leq c \triangleleft_{i} c \leq b$. Note that, by (SB3), $c \triangleleft_{i} c$ means that $c \in L\left(\triangleleft_{i}\right)$ is complemented in $L$ with complement in $L\left(\triangleleft_{j}\right)(j \neq i)$.

\section{Quasi-proximities and quasi-uniformities: total boundedness}

To finish we show, in analogy with the spatial case or the symmetric case (see $[5,7,9,12,13]$ ), that the category QPFrm is isomorphic to the full subcategory TBQUFm of QUFrm of all totally bounded quasi-uniform frames.

First, we need a few basic facts about paircovers. Let $C$ be a paircover of the frame $L$. Set

$$
C_{s}=\left\{\left(c_{1}, c_{2}\right) \in C \mid c_{1} \wedge c_{2} \neq 0\right\} .
$$

Lemma 4.1. Let $C, D$ be paircovers of the frame $L$. Then:

(1) $C_{s}$ is a strong paircover of $L$.

(2) $(C \wedge D)_{s} \leq C_{s} \wedge D_{s}$.

(3) $C^{*} \leq D \rightarrow\left(C_{s}\right)^{*} \leq D_{s}$.

(4) If $h: L \rightarrow M$ is a frame homomorphism, then $(h[C])_{s} \leq h\left[C_{s}\right]$. 
Proof: (1) and (2) are obvious.

(3) Let $\left(c_{1}, c_{2}\right) \in C$ with $c_{1} \wedge c_{2} \neq 0$. Since $\operatorname{st}_{i}\left(c_{i}, C_{s}\right) \leq \operatorname{st}_{i}\left(c_{i}, C\right) \leq d_{i}$ $(i=1,2)$ for some $\left(d_{1}, d_{2}\right) \in D$ and $c_{1} \wedge c_{2} \leq \operatorname{st}_{1}\left(c_{1}, C_{s}\right) \wedge \mathrm{st}_{2}\left(c_{2}, C_{s}\right) \leq d_{1} \wedge d_{2}$, then $\left(d_{1}, d_{2}\right) \in D_{s}$.

(4) Suppose $h\left(c_{1}\right) \wedge h\left(c_{2}\right) \neq 0$ for $\left(c_{1}, c_{2}\right) \in C$. Then $h\left(c_{1} \wedge c_{2}\right) \neq 0$, hence $c_{1} \wedge c_{2} \neq 0$ and consequently $\left(c_{1}, c_{2}\right) \in C_{s}$. Hence $\left(h\left(c_{1}\right), h\left(c_{2}\right)\right) \in h\left[C_{s}\right]$.

Lemma 4.2. Let $\mathcal{U}$ be an admissible filter of paircovers of $L$ with property (QU3), and let $\mathcal{U}_{s}$ be the filter of paircovers of $L$ that has $\left\{C_{s} \mid C \in \mathcal{U}\right\}$ as subbasis. Then $\left(L, \mathcal{U}_{s}\right)$ is a quasi-uniform frame.

Proof: $\mathcal{U}_{s}$ is a filter of paircovers of $L$ which by Lemma 4.1 satisfies the requirements (QU1)-(QU3). Since $\mathcal{U} \subseteq \mathcal{U}_{s}$, then $L_{i}(\mathcal{U}) \subseteq L_{i}\left(\mathcal{U}_{s}\right)(i=1,2)$. Hence $\left(L, L_{1}\left(\mathcal{U}_{s}\right), L_{2}\left(\mathcal{U}_{s}\right)\right)$ is also a biframe and $\mathcal{U}_{s}$ is admissible.

Let $\left(L, \triangleleft_{1}, \triangleleft_{2}\right)$ be a quasi-proximal frame. For any $a \triangleleft_{1} b$ we define

$$
C_{a, b}^{1}=\left\{\left(1, a^{*}\right),(b, 1)\right\}
$$

and for $a \triangleleft_{2} b$ we define

$$
C_{a, b}^{2}=\left\{\left(a^{*}, 1\right),(1, b)\right\} .
$$

Of course, $C_{a, b}^{1}$ and $C_{a, b}^{2}$ are paircovers of $L$.

Proposition 4.3. The filter $\mathcal{U}$ of paircovers of $L$ which has as subbasis the family of paircovers $\left\{C_{a, b}^{i} \mid a \triangleleft_{i} b, i=1,2\right\}$ satisfies the conditions of the preceding lemma.

Proof: Let $a \triangleleft_{1} b$. Use (SB4) to select $c_{1}, c_{2} \in L\left(\triangleleft_{1}\right)$ such that $a \triangleleft_{1} c_{1} \triangleleft_{1} c_{2} \triangleleft_{1} b$ and consider $C=C_{a, c_{1}}^{1} \wedge C_{c_{1}, c_{2}}^{1} \wedge C_{c_{2}, b}^{1}$. Then

$$
C=\left\{\left(c_{1}, 1\right),\left(c_{1}, c_{1}^{*}\right),\left(c_{1}, c_{2}^{*}\right),\left(c_{2}, a^{*}\right),\left(c_{2}, c_{2}^{*}\right),\left(b, c_{1}^{*}\right),\left(1, c_{2}^{*}\right)\right\} .
$$

Since

$$
\mathrm{st}_{1}\left(c_{1}, C\right) \leq b, \quad \mathrm{st}_{1}\left(c_{2}, C\right) \leq b, \quad \mathrm{st}_{2}\left(c_{1}^{*}, C\right) \leq a^{*} \quad \text { and } \quad \mathrm{st}_{2}\left(c_{2}^{*}, C\right) \leq a^{*},
$$

we have $C^{*} \leq C_{a, b}^{1}$. Similarly, for $a \triangleleft_{2} b$ and $a \triangleleft_{2} c_{1} \triangleleft_{2} c_{2} \triangleleft_{2} b$,

$$
\left(C_{a, c_{1}}^{2} \wedge C_{c_{1}, c_{2}}^{2} \wedge C_{c_{2}, b}^{2}\right)^{*} \leq C_{a, b}^{2} .
$$

This shows that $\mathcal{U}$ satisfies (QU3). In order to prove the admissibility of $\mathcal{U}$ it suffices to show that $a \triangleleft_{i} b$ implies $a \triangleleft_{i}^{\mathcal{U}} b$ which is obvious since $a \triangleleft_{i} b$ implies $\mathrm{st}_{i}\left(a, C_{a, b}^{i}\right) \leq b$. 
Hence the corresponding $\mathcal{U}_{s}$ given by the lemma is a quasi-uniformity on $L$ (which is of course totally bounded). We shall denote it by $\mathcal{U}_{F}\left(L, \triangleleft_{1}, \triangleleft_{2}\right)$.

Proposition 4.4. The correspondence $\left(L, \triangleleft_{1}, \triangleleft_{2}\right) \rightsquigarrow\left(L, \mathcal{U}_{F}\left(L, \triangleleft_{1}, \triangleleft_{2}\right)\right)$ determines a concrete functor $\Phi:$ QPFrm $\rightarrow$ TBQUFrm.

Proof: It remains to check that for any quasi-proximal map

$$
\begin{gathered}
h:\left(L, \triangleleft_{1}^{L}, \triangleleft_{2}^{L}\right) \rightarrow\left(M, \triangleleft_{1}^{M}, \triangleleft_{2}^{M}\right), \\
h:\left(L, \mathcal{U}_{F}\left(L, \triangleleft_{1}^{L}, \triangleleft_{2}^{L}\right)\right) \rightarrow\left(M, \mathcal{U}_{F}\left(M, \triangleleft_{1}^{M}, \triangleleft_{2}^{M}\right)\right)
\end{gathered}
$$

is a quasi-uniform map. By Lemma 4.1(4) it suffices to show that $h\left[C_{a, b}^{i}\right] \in$ $\mathcal{U}_{F}\left(M, \triangleleft_{1}^{M}, \triangleleft_{2}^{M}\right)$ whenever $a \triangleleft_{i} b(i=1,2)$.

Suppose $a \triangleleft_{i} b$ and apply (SB4) to select $c \in L\left(\triangleleft_{i}\right)$ such that $a \triangleleft_{i} c \triangleleft_{i} b$. By (SB3), $a^{*} \vee c=1$, which in turn implies that $h(c)^{*} \leq h\left(a^{*}\right)$ :

$a^{*} \vee c=1 \Rightarrow h\left(a^{*}\right) \vee h(c)=1 \Rightarrow h(c)^{*}=h(c)^{*} \wedge\left(h\left(a^{*}\right) \vee h(c)\right)=h(c)^{*} \wedge h\left(a^{*}\right)$

Hence

$$
C_{h(c), h(b)}^{1}=\left\{\left(1, h(c)^{*}\right),(h(b), 1)\right\}
$$

refines $h\left[C_{a, b}^{1}\right]=\left\{\left(1, h\left(a^{*}\right),(h(b), 1)\right\}\right.$ and

$$
C_{h(c), h(b)}^{2}=\left\{\left(h(c)^{*}, 1\right),(1, h(b))\right\}
$$

refines $h\left[C_{a, b}^{2}\right]=\left\{\left(h\left(a^{*}\right), 1\right),(1, h(b))\right\}$.

On the other hand, going back to Proposition 2.5, we have:

Proposition 4.5. The correspondence $(L, \mathcal{U}) \rightsquigarrow\left(L, \triangleleft_{1}^{\mathcal{U}}, \triangleleft_{2}^{\mathcal{U}}\right)$ determines a concrete functor $\Psi:$ TBQUFrm $\rightarrow$ QPFrm.

Proof: Let $h:(L, \mathcal{U}) \rightarrow(M, \mathcal{V})$ be a quasi-uniform map. We need to show that

$$
h:\left(L, \triangleleft_{1}^{\mathcal{U}}, \triangleleft_{2}^{\mathcal{U}}\right) \rightarrow\left(M, \triangleleft_{1}^{\mathcal{V}}, \triangleleft_{2}^{\mathcal{V}}\right)
$$

is a quasi-proximal map, that is, $a \triangleleft_{i}^{\mathcal{U}} b$ implies $h(a) \triangleleft_{i}^{\mathcal{V}} h(b)$. But $a \triangleleft_{1}^{\mathcal{U}} b$ means that $\operatorname{st}_{i}(a, U) \leq b$ for some $U \in \mathcal{U}$ and, by the statement (6) in 1.3.1, st $_{i}(h(a), h[U]) \leq h(b)$. Since $h[U] \in \mathcal{V}$ this makes $h(a) \triangleleft_{i}^{\mathcal{V}} h(b)$, as required.

Theorem 4.6. The functors $\Phi$ and $\Psi$ constitute a concrete isomorphism between QPFrm and TBQUFrm. 
Proof: We want to show that $\Psi \Phi=\operatorname{Id}_{\text {QPFrm }}$ and $\Phi \Psi=\operatorname{Id}_{\text {TBQUFrm }}$. After 4.4 and 4.5 there is nothing left to prove for morphisms.

Now for the objects. We have

$$
\begin{aligned}
& \Phi \Psi((L, \mathcal{U}))=\Phi\left(\left(L, \triangleleft_{1}^{\mathcal{U}}, \triangleleft_{2}^{\mathcal{U}}\right)\right)=\left(L, \mathcal{U}_{F}\left(L, \triangleleft_{1}^{\mathcal{U}}, \triangleleft_{2}^{\mathcal{U}}\right)\right) \quad \text { and } \\
& \Psi \Phi\left(\left(L, \triangleleft_{1}, \triangleleft_{2}\right)\right)=\Psi\left(\left(L, \mathcal{U}_{F}\left(L, \triangleleft_{1}, \triangleleft_{2}\right)\right)\right)=\left(L, \triangleleft_{1}^{\mathcal{U}_{F}\left(L, \triangleleft_{1}, \triangleleft_{2}\right)}, \triangleleft_{2}^{\mathcal{U}_{F}\left(L, \triangleleft_{1}, \triangleleft_{2}\right)}\right),
\end{aligned}
$$

so that we need to prove that
(a) $\mathcal{U}_{F}\left(L, \triangleleft_{1}^{\mathcal{U}}, \triangleleft_{2}^{\mathcal{U}}\right)=\mathcal{U}$ and
(b) $\triangleleft_{i}^{\mathcal{U}_{F}\left(L, \triangleleft_{1}, \triangleleft_{2}\right)}=\triangleleft_{i}$ for $i=1,2$.

(a) Let $C_{a, b}^{i}$ be a subbasic paircover of $\mathcal{U}_{F}\left(L, \triangleleft_{1}^{\mathcal{U}}, \triangleleft_{2}^{\mathcal{U}}\right)$. Then $a \triangleleft_{i}^{\mathcal{U}} b$, that is, st $_{i}(a, U) \leq b$ for some $U \in \mathcal{U}$.

Suppose $i=1$ and let $\left(u_{1}, u_{2}\right) \in U$. If $u_{2} \wedge a \neq 0$ then $u_{1} \leq \operatorname{st}_{1}(a, U) \leq b$ and $\left(u_{1}, u_{2}\right) \leq(b, 1)$; otherwise, $u_{2} \leq a^{*}$ so $\left(u_{1}, u_{2}\right) \leq\left(1, a^{*}\right)$. Hence $U \leq$ $C_{a, b}^{1}$ which shows that $C_{a, b}^{1} \in \mathcal{U}$. The case $i=2$ proceeds similarly. Thus $\mathcal{U}_{F}\left(L, \triangleleft_{1}^{\mathcal{U}}, \triangleleft_{2}^{\mathcal{U}}\right) \subseteq \mathcal{U}$.

Now let $U \in \mathcal{U}$ and select a strong paircover $V \in \mathcal{U}$ such that $V^{*} \leq U$. Since $\mathcal{U}$ is totally bounded, there is a finite $F \subseteq V$ such that $F$ is still a paircover:

$$
F=\left\{\left(c_{1}, d_{1}\right),\left(c_{2}, d_{2}\right) \ldots,\left(c_{n}, d_{n}\right)\right\}, \quad c_{i} \wedge d_{i} \neq 0, \quad \bigvee_{i=1}^{n}\left(c_{i} \wedge d_{i}\right)=1
$$

Since $\left(\operatorname{st}_{1}\left(c_{i}, V\right), \mathrm{st}_{2}\left(d_{i}, V\right)\right) \leq\left(u_{i}, v_{i}\right)$ for some $\left(u_{i}, v_{i}\right) \in U(i=1,2, \ldots, n)$, it follows that $c_{i} \triangleleft_{1}^{\mathcal{U}} u_{i}$ and $d_{i} \triangleleft_{2}^{\mathcal{U}} v_{i}$. It suffices now to show that for

$$
C:=C_{c_{1}, u_{1}}^{1} \wedge C_{c_{2}, u_{2}}^{1} \wedge \cdots \wedge C_{c_{n}, u_{n}}^{1} \wedge C_{d_{1}, v_{1}}^{2} \wedge C_{d_{2}, v_{2}}^{2} \wedge \cdots \wedge C_{d_{n}, v_{n}}^{2},
$$

the corresponding strong paircover $C_{s}$ (which belongs to $\mathcal{U}_{F}\left(L, \triangleleft_{1}^{\mathcal{U}}, \triangleleft_{2}^{\mathcal{U}}\right)$ ) is a refinement of $U$.

Any element $(x, y)$ in $C$ is of the form

$$
\left(u_{i_{1}} \wedge u_{i_{2}} \wedge \cdots \wedge u_{i_{k}} \wedge d_{j_{1}^{\prime}}^{*} \wedge d_{j_{2}^{\prime}}^{*} \wedge \cdots \wedge d_{j_{n-l}^{\prime}}^{*}, v_{j_{1}} \wedge v_{j_{2}} \wedge \cdots \wedge v_{j_{l}} \wedge c_{i_{1}^{\prime}}^{*} \wedge c_{i_{2}^{\prime}}^{*} \wedge \cdots \wedge c_{i_{n-k}^{\prime}}^{*}\right)
$$

where

$$
I=\left\{i_{1}, i_{2}, \ldots, i_{k}\right\}, \quad I^{\prime}=\left\{i_{1}^{\prime}, i_{2}^{\prime}, \ldots, i_{n-k}^{\prime}\right\}
$$

and

$$
J=\left\{j_{1}, j_{2}, \ldots, j_{l}\right\}, \quad J^{\prime}=\left\{j_{1}^{\prime}, j_{2}^{\prime}, \ldots, j_{n-l}^{\prime}\right\}
$$

are partitions of $\bar{n}=\{1,2, \ldots, n\}$. 
If $I \cap J \neq \varnothing$ then $(x, y) \leq\left(u_{\alpha}, v_{\alpha}\right) \in U$ for $\alpha \in I \cap J$. Otherwise, if $I \cap J=\varnothing$ then $I^{\prime} \cup J^{\prime}=\bar{n}$ (and $k+l=n$ ). Since

$$
(x, y) \leq\left(d_{j_{1}^{\prime}}^{*} \wedge d_{j_{2}^{\prime}}^{*} \wedge \cdots \wedge d_{j_{n-l}^{\prime}}^{*}, c_{i_{1}^{\prime}}^{*} \wedge c_{i_{2}^{\prime}}^{*} \wedge \cdots \wedge c_{i_{n-k}^{\prime}}^{*}\right)
$$

this in turn implies that

$$
x \wedge y \leq \bigwedge_{i=1}^{n}\left(c_{i}^{*} \vee d_{i}^{*}\right)
$$

But

$$
\bigvee_{i=1}^{n}\left(c_{i} \wedge d_{i}\right)=1 \Rightarrow \bigwedge_{i=1}^{n}\left(c_{i} \wedge d_{i}\right)^{*}=0 \Rightarrow \bigwedge_{i=1}^{n}\left(c_{i}^{*} \vee d_{i}^{*}\right)=0
$$

Hence $x \wedge y=0$ and $(x, y) \notin C_{s}$.

(b) If $a \triangleleft_{i} b$ then $C_{a, b}^{i} \in \mathcal{U}_{F}\left(L, \triangleleft_{1}, \triangleleft_{2}\right)$. Since $\operatorname{st}_{i}\left(a, C_{a, b}^{i}\right) \leq b$ we have

$$
a \triangleleft_{i}^{\mathcal{U}_{F}\left(L, \triangleleft_{1}, \triangleleft_{2}\right)} b .
$$

Conversely, suppose for some $U \in \mathcal{U}_{F}\left(L, \triangleleft_{1}, \triangleleft_{2}\right)$ we have st $_{i}(a, U) \leq b$ $(i=1$ or $i=2)$. Our aim is to show that $a \triangleleft_{i} b$. We may assume that

$$
U=C_{a_{1}^{1}, b_{1}^{1}}^{1} \wedge C_{a_{2}^{1}, b_{2}^{1}}^{1} \wedge \cdots \wedge C_{a_{n}^{1}, b_{n}^{1}}^{1} \wedge C_{a_{1}^{2}, b_{1}^{2}}^{2} \wedge C_{a_{2}^{2}, b_{2}^{2}}^{2} \wedge \cdots \wedge C_{a_{m}^{2}, b_{m}^{2}}^{2}
$$

where $a_{\alpha}^{1} \triangleleft_{1} b_{\alpha}^{1}$ for $\alpha=1,2, \ldots, n$ and $a_{\beta}^{2} \triangleleft_{2} b_{j}^{2}$ for $\beta=1,2, \ldots m$. Any element $\left(u_{1}, u_{2}\right)$ in $U$ is of the form

$$
\begin{aligned}
\left(b_{\alpha_{1}}^{1} \wedge b_{\alpha_{2}}^{1} \wedge \cdots \wedge b_{\alpha_{k}}^{1} \wedge\left(a_{\beta_{1}^{\prime}}^{2}\right)^{*}\right. & \wedge\left(a_{\beta_{2}^{\prime}}^{2}\right)^{*} \wedge \cdots \wedge\left(a_{\beta_{m-l}^{\prime}}^{2}\right)^{*} \\
& \left.b_{\beta_{1}}^{2} \wedge b_{\beta_{2}}^{2} \wedge \cdots \wedge b_{\beta_{l}}^{2} \wedge\left(a_{\alpha_{1}^{\prime}}^{1}\right)^{*} \wedge\left(a_{\alpha_{2}^{\prime}}^{1}\right)^{*} \wedge \cdots \wedge\left(a_{\alpha_{n-k}^{\prime}}^{1}\right)^{*}\right)
\end{aligned}
$$

where

$$
A=\left\{\alpha_{1}, \alpha_{2}, \ldots, \alpha_{k}\right\}, \quad A^{\prime}=\left\{\alpha_{1}^{\prime}, \alpha_{2}^{\prime}, \ldots, \alpha_{n-k}^{\prime}\right\}
$$

is a partition of $\bar{n}=\{1,2, \ldots, n\}$ and

$$
B=\left\{\beta_{1}, \beta_{2}, \ldots, \beta_{l}\right\}, \quad B^{\prime}=\left\{\beta_{1}^{\prime}, \beta_{2}^{\prime}, \ldots, \beta_{m-l}^{\prime}\right\}
$$

is a partition of $\bar{m}=\{1,2, \ldots, m\}$. Select $c_{\alpha}^{1}, d_{\alpha}^{1} \in L$ such that

$$
a_{\alpha}^{1} \triangleleft_{1} c_{\alpha}^{1} \triangleleft_{1} d_{\alpha}^{1} \triangleleft_{1} b_{\alpha}^{1} \quad \text { for every } \alpha \in \bar{n}
$$

and $c_{\beta}^{2}, d_{\beta}^{2} \in L$ such that

$$
a_{\beta}^{2} \triangleleft_{2} c_{\beta}^{2} \triangleleft_{2} d_{\beta}^{2} \triangleleft_{2} b_{\beta}^{2} \quad \text { for every } \beta \in \bar{m} .
$$


By (SB5), $\left(c_{\beta}^{2}\right)^{*} \triangleleft_{1}\left(a_{\beta}^{2}\right)^{*}$ for every $\beta \in \bar{m}$ and $\left(c_{\alpha}^{1}\right)^{*} \triangleleft_{2}\left(a_{\alpha}^{1}\right)^{*}$ for every $\alpha \in \bar{n}$. Suppose $\left(u_{1}, u_{2}\right) \in U$ is such that

$$
u_{1}=\bigwedge_{\alpha \in A} b_{\alpha}^{1} \wedge \bigwedge_{\beta \in B^{\prime}}\left(a_{\beta}^{2}\right)^{*} \quad \text { and } \quad u_{2}=\bigwedge_{\beta \in B} b_{\beta}^{2} \wedge \bigwedge_{\alpha \in A^{\prime}}\left(a_{\alpha}^{1}\right)^{*},
$$

with $A \cup A^{\prime}=\bar{n}, A \cap A^{\prime}=\varnothing, B \cup B^{\prime}=\bar{m}, B \cap B^{\prime}=\varnothing$. Then set

$$
\widetilde{u}_{1}=\bigwedge_{\alpha \in A} d_{\alpha}^{1} \wedge \bigwedge_{\beta \in B^{\prime}}\left(c_{\beta}^{2}\right)^{*} \quad \text { and } \quad \widetilde{u}_{2}=\bigwedge_{\beta \in B} d_{\beta}^{2} \wedge \bigwedge_{\alpha \in A^{\prime}}\left(c_{\alpha}^{1}\right)^{*} .
$$

Clearly $\widetilde{u}_{1} \triangleleft_{1} u_{1}$ and $\widetilde{u}_{2} \triangleleft_{2} u_{2}$. Therefore $u_{1}^{*} \triangleleft_{2}\left(\widetilde{u}_{1}\right)^{*}$ and $u_{2}^{*} \triangleleft_{1}\left(\widetilde{u}_{2}\right)^{*}$.

Let $j \in\{1,2\}, j \neq i$, and

$$
U_{1}=\left\{\left(u_{1}, u_{2}\right) \in U \mid u_{j} \wedge a=0\right\}, \quad U_{2}=\left\{\left(u_{1}, u_{2}\right) \in U \mid u_{j} \wedge a \neq 0\right\} .
$$

This is a partition of $U$. Now, by the first De Morgan law (which holds in any frame),

$$
a \leq \bigwedge\left\{u_{j}^{*} \mid\left(u_{1}, u_{2}\right) \in U_{1}\right\}=\left(\bigvee\left\{u_{j} \mid\left(u_{1}, u_{2}\right) \in U_{1}\right\}\right)^{*} .
$$

Since $U$ is finite and $\widetilde{u}_{j} \triangleleft_{j} u_{j}$, we have

$$
\bigvee\left\{\widetilde{u}_{j} \mid\left(u_{1}, u_{2}\right) \in U_{1}\right\} \triangleleft_{j} \bigvee\left\{u_{j} \mid\left(u_{1}, u_{2}\right) \in U_{1}\right\}
$$

and then by (SB5)

$$
\left(\bigvee\left\{u_{j} \mid\left(u_{1}, u_{2}\right) \in U_{1}\right\}\right)^{*} \triangleleft_{i}\left(\bigvee\left\{\widetilde{u}_{j} \mid\left(u_{1}, u_{2}\right) \in U_{1}\right\}\right)^{*} .
$$

But $\left\{\left(\widetilde{u}_{1}, \widetilde{u}_{2}\right) \mid\left(u_{1}, u_{2}\right) \in U\right\}$ is a paircover of $L$ (it coincides with the paircover

$$
\left.C_{c_{1}^{1}, d_{1}^{1}}^{1} \wedge C_{c_{2}^{1}, d_{2}^{1}}^{1} \wedge \cdots \wedge C_{c_{n}^{1}, d_{n}^{1}}^{1} \wedge C_{c_{1}^{2}, d_{1}^{2}}^{2} \wedge C_{c_{2}^{2}, d_{2}^{2}}^{2} \wedge \cdots \wedge C_{c_{m}^{2}, d_{m}^{2}}^{2}\right) .
$$

This means that

$$
1=\bigvee_{\left(u_{1}, u_{2}\right) \in U}\left(\widetilde{u}_{1} \wedge \widetilde{u}_{2}\right) \leq \bigvee\left\{\widetilde{u}_{j} \mid\left(u_{1}, u_{2}\right) \in U_{1}\right\} \vee \bigvee\left\{\widetilde{u}_{i} \mid\left(u_{1}, u_{2}\right) \in U_{2}\right\}
$$

which immediately implies that

$$
\begin{aligned}
\left(\bigvee\left\{\widetilde{u}_{j} \mid\left(u_{1}, u_{2}\right) \in U_{1}\right\}\right)^{*} & \leq \bigvee\left\{\widetilde{u}_{i} \mid\left(u_{1}, u_{2}\right) \in U_{2}\right\} \\
& \leq \bigvee\left\{u_{i} \mid\left(u_{1}, u_{2}\right) \in U_{2}\right\}=\operatorname{st}_{i}(a, U) \leq b
\end{aligned}
$$

By (4.6.1), (4.6.2) and (4.6.3) we have $a \triangleleft_{i} b$ as desired. 
Remark 4.7. The dual adjoint situation between quasi-uniform spaces and quasi-uniform frames established in [15] by functors

$$
\Omega: \text { QUnif } \rightarrow \text { QUFrm and } \Sigma: \text { QUFrm } \rightarrow \text { QUnif }
$$

restricts immediately to a dual adjunction

$$
\text { QProx } \leftrightarrows \text { QPFrm }
$$

between the categories of quasi-proximal spaces and quasi-proximal frames (since for each totally bounded quasi-uniform space $(X, \mu), \Omega(X, \mu)$ is a totally bounded quasi-uniform frame and for each totally bounded quasiuniform frame $(L, \mathcal{U})$, the quasi-uniform space $\Sigma(L, \mathcal{U})$ is also totally bounded).

\section{References}

[1] B. Banaschewski, Compactification of frames, Math. Nachr. 149 (1990) 105-116.

[2] B. Banaschewski, G. C. L. Brümmer and K. A. Hardie, Biframes and bispaces, Quaest. Math. 6 (1983) 13-25.

[3] D. Doitchinov, Some reflections on quasi-uniform frames, in: Topology with Applications (Szekszàrd, Hungary, 1993), vol. 4, pp. 151-158, Bolyai Society, Math. Studies, 1995.

[4] C. H. Dowker, Mappings of proximity structures, in: General Topology and its Relation to Modern Analysis and Algebra (Proc. Sympos., Prague, 1961), pp. 139-141, Academic Press, New York; Publ. House, Czech. Acad. Sci., Prague, 1962.

[5] P. Fletcher and W. Hunsaker, Totally bounded uniformities for frames, Topology Proc. 17 (1992) 59-69.

[6] P. Fletcher and W. Lindgren, Quasi-uniform Spaces, Marcel Dekker, New York, 1982.

[7] J. Frith, Structured Frames, PhD thesis, University of Cape Town, 1987.

[8] J. Frith and A. Schauerte, The Samuel compactification for quasi-uniform biframes, Topol. Appl. 156 (2009) 2116-2122.

[9] W. Hunsaker and J. Picado, A note on total boundedness, Acta Math. Hungarica 88 (2000) 25-34.

[10] P. T. Johnstone, Stone Spaces, Cambridge Studies in Advanced Math., vol. 3, Cambridge Univ. Press, Cambridge, 1982.

[11] H.-P. Künzi, Nonsymmetric distances and their associated topologies: About the origins of basic ideas in the area of asymmetric topology, in: C. E. Aull, R. Lowen (Eds.), Handbook of the History of General Topology, vol. 3, pp. 853-968, Kluwer, 2001.

[12] S. A. Naimpally and B. D. Warrack, Proximity Spaces, Cambridge Tracts in Math. and Math. Phys., vol. 59, Cambridge University Press, Cambridge, 1970.

[13] J. Picado, Weil Entourages in Pointfree Topology, PhD Thesis, University of Coimbra, 1995.

[14] J. Picado, Structured frames by Weil entourages, Appl. Categ. Structures 8 (2000) 351366.

[15] J. Picado and A. Pultr, Cover quasi-uniformities in frames, preprint KAM-DIMATIA 2009-947, submitted for publication. 
[16] J. Picado, A. Pultr and A. Tozzi, Locales, in: M. C. Pedicchio, W. Tholen (Eds.), Categorical Foundation - Special Topics in Order, Algebra and Sheaf Theory, Encyclopedia of Mathematics and its Applications, vol. 97, pp. 49-101, Cambridge Univ. Press, Cambridge, 2004.

[17] A. Pultr, Frames, in: M. Hazewinkel (Ed.), Handbook of Algebra, vol. 3, pp. 791-858, Elsevier, 2003.

[18] A. Schauerte, Biframes, PhD thesis, McMaster University, 1992.

[19] A. Schauerte, Biframe compactifications, Comment. Math. Univ. Carolin. 34 (1993) $567-574$.

Jorge PicAdo

CMUC, Department of Mathematics, University of Coimbra, 3001-454 Coimbra, Portugal

E-mail address: picado@mat.uc.pt

$U R L$ : www.mat.uc.pt/ picado

Aleš Pultr

Department of Applied Mathematics and iti, MFF, Charles University, Malostranské NÁM. 24, 11800 Praha 1, CZech Republic

E-mail address: pultr@kam.ms.mff.cuni.cz 